\title{
Connecting with Users though Interactive Prototyping: Understanding User Behaviour through Building the Black Box and its Electronic Innards
}

\author{
Andre Murnieks \\ University of Notre Dame \\ Notre Dame, Indiana, \\ USA \\ andre.murnieks@nd.edu
}

\section{MANUFACTURED EXPERIENCE}

Interaction design creates new challenges for the researcher, educator and student. Certainly, the "look" of an interaction system falls within the purview of visual communication design; however, the correlating area of "feel" is fairly new territory for designers. The term "feel" acknowledges that information being relayed extends beyond the realm of vision; the most interactive experiences depend on multiple senses and feedback processes: gaze, aural, gesture, touch, haptic, biometrics (heart rate, pulse...).

In turn, the senses and feedback are processed though perceptual and cognitive systems of the nervous system and brain (Ware 2008). The behavioural responses to an interactive system are dependent on both physiological and psychological dimensions; this is how humans interact. However, interaction design, as a discipline, is a manufactured experience between human and machine or human-to-human(s) through machine.

\section{THE ESSENCE OF INTERACTION}

Clicking through on-screen prototypes helps designers anticipate the user's behaviour and response. Yet, we are often surprised with the results when we test with our intended user group. The desktop computer provides an interaction paradigm that is narrow in scope (point and click), and the user experience is limited to what is prescribed by the operating system. This restraint also disconnects the designer from the form factor, the underlying circuitry and the programming necessary to make a desired computing environment possible. Without a flexible, platform, creating accurate and multi-sensory simulations is difficult. However, it is possible to explore the essence of pure interaction design with a prototyping platform like Arduino. Using an off-theshelf programmable microprocessor, the student researcher can build, program, and test a simple interactive widget.

\section{FOUNDATIONS FOR INTERACTION DESIGN}

Just as design educators feel it is imperative to understand the processes behind print and production, this is a foundational exercise for interaction design allowing for a deep understanding of human interaction with respect to the programmable logic in electronic devices. The purity of this exercise can lead to an unexpected result: a sculptural and engaging object of mystery and delight. Rather than a demonstration of Arduino, the making of the fortune telling device, Oracle In $A$ Box, reveals the synergy of visual communication, industrial design, advanced 3D fabrication and programming logic to create perceived awareness in an obelisk of acrylic, sound and light.

\section{CONCEPT AS APPLIED RESEARCH}

To quote from Murnieks (2013):
"Introducing the state-of-the-art in freestanding, prognosticatory devices. With version 2.0 of Oracle In A Box, the future is literally at your fingertips. The Oracle utilizes the latest in electro-biometric sensory to deduce (actually induce) your probable outlook with a light touch of the fingers on the advanced, ergonomic control interface. Within just a few seconds, the 
Oracle will respond with its prophecy in the form of a custom tailored sound and light pattern. Will your fortune be dark and foreboding? Or will it be bright and cheery? Only your touch will tell."

\section{REFERENCES}

Murnieks, A. (2013) The Making of Oracle In A Box v2.0. Vimeo. https://vimeo.com/81412178

(accessed 18 May 2014).

Ware, C. (2008) Visual Thinking for Design. Burlington, Mass.: Morgan Kaufmann; Amsterdam: Elsevier Science \& Technology.

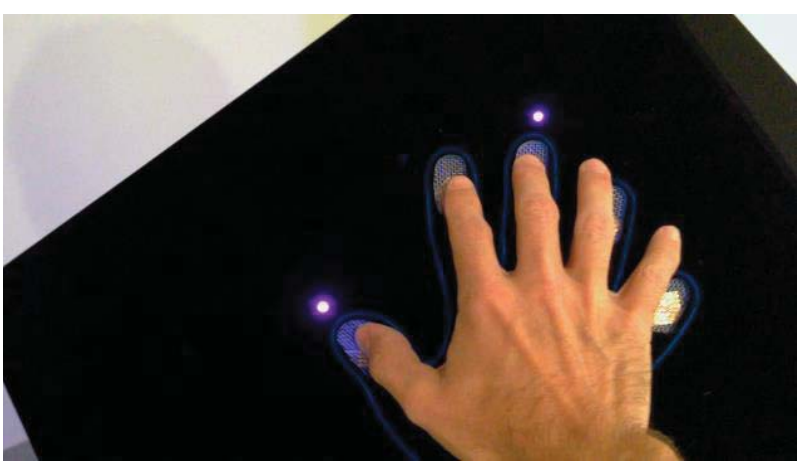

Figure 1: Underlying coordinated complexity is responsible for perceived simplicity

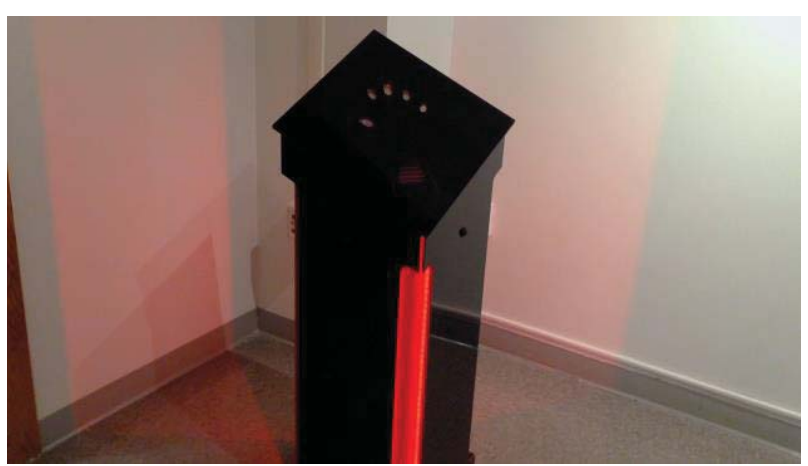

Figure 2: "Oracle In A Box" illuminated in red

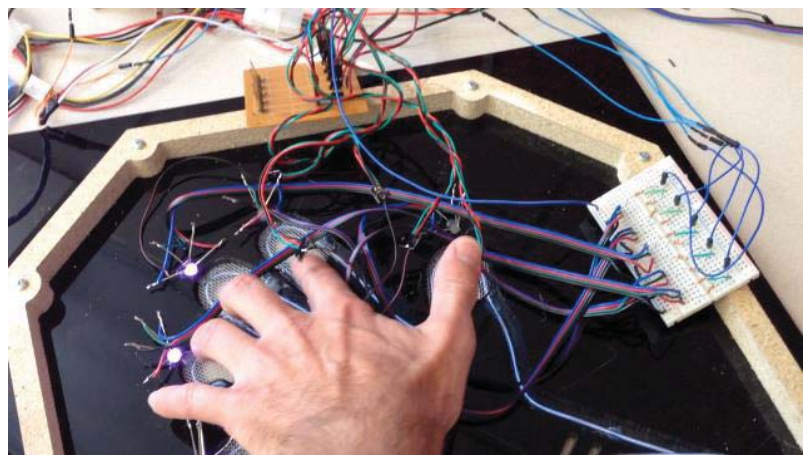

Figure 3: Circuitry for light, motion and touch

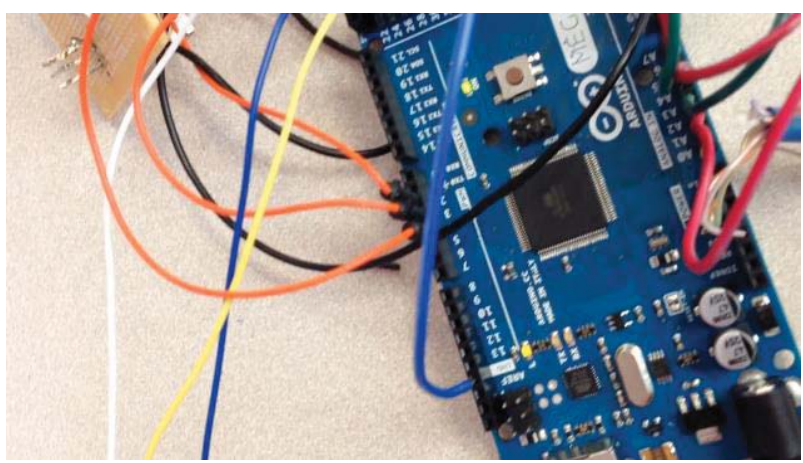

Figure 4: Programmable microprocessor controls interaction through user actions, reactions, and feedback

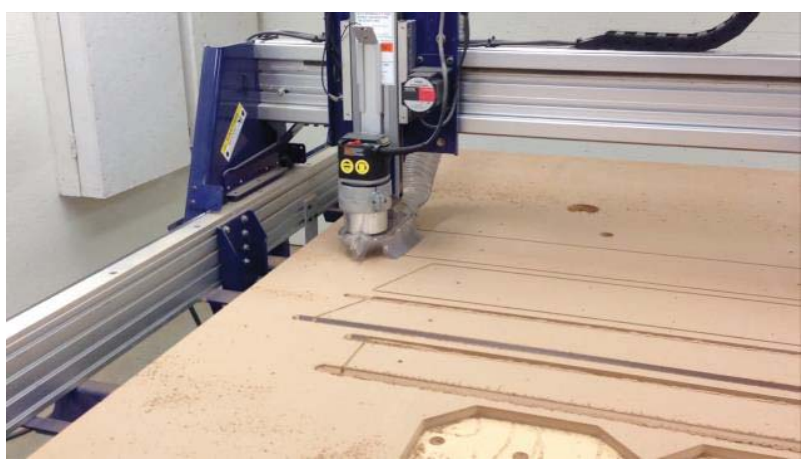

Figure 5: CNC router cutting acrylic of product form 